\title{
Incidental finding of papillary thyroid carcinoma on CT examination of mandibular lesion: Case report
}

\author{
IRFAN SUGIANTO $^{1,2}$, YOSHINOBU YANAGI ${ }^{3}$, HIDENOBU KONOUCHI $^{1}$, MIKI HISATOMI $^{3}$, \\ SHUNSUKE OKADA ${ }^{1}$, BABATUNDE O. BAMGBOSE ${ }^{4}$ and JUNICHI ASAUMI ${ }^{1,3}$ \\ ${ }^{1}$ Department of Oral and Maxillofacial Radiology, Okayama University Graduate School of Medicine, Dentistry and \\ Pharmaceutical Sciences, Okayama 700-8558, Japan; ${ }^{2}$ Department of Oral Radiology Faculty of Dentistry, \\ Hasanuddin University, Makassar 90245, Indonesia; ${ }^{3}$ Department of Oral Diagnosis and \\ Dentomaxillofacial Radiology, Okayama University Hospital; ${ }^{4}$ Department of Oral and \\ Maxillofacial Radiology, Okayama University, Okayama 700-8530, Japan
}

Received June 13,2017; Accepted September 28, 2017

DOI: $10.3892 / \mathrm{mco} .2017 .1478$

\begin{abstract}
The number of significant incidental findings identified on radiographic examinations has increased. In total, 169 patients, 87 males and 82 females, with a mean age of 67.3 years (range, 17-92 years), as well as 65 patients (38.5\%), $43(66.2 \%)$ female and $22(33.8 \%)$ male had abnormal findings in the thyroid for one year in our institution. Thyroid nodules are the most common incidental findings on oral and maxillofacial evaluations. Computed tomography (CT) and magnetic resonance imaging (MRI) examination are frequently used to detect thyroid abnormality. The present study reported a case of papillary thyroid carcinoma (PTC) detected following CT and MRI examination of a mandibular lesion. The CT examination of the oral and maxillofacial region included the thyroid, which must be examined carefully to detect various diseases, including PTC. Collaboration between radiologists and clinicians is important to manage these incidental findings.
\end{abstract}

\section{Introduction}

Imaging technology is continuously improving, and advanced diagnostic imaging modalities such as high resolution ultrasonography (US), computed tomography (CT) and magnetic resonance imaging (MRI) can obtain images of wider areas over shorter times. Along with this technical advancement, the detection of unexpected lesions has increased (1). Such findings are referred to as 'incidental findings'. Incidental findings

Correspondence to: Dr Irfan Sugianto, Department of Oral and Maxillofacial Radiology, Okayama University Graduate School of Medicine, Dentistry and Pharmaceutical Sciences, 5-1 Shikata-Cho, 2 Chome, Kita-Ku, Okayama-Shi, Okayama 700-8558, Japan

E-mail: pp162rsv@s.okayama-u.ac.jp

Key words: incidental findings, computed tomography, thyroid nodule, papillary thyroid carcinoma include masses (often called 'incidentalomas') or anatomic malformations. Incidental thyroid nodular lesions are common and were first identified on CT (2-4).

The most common type of thyroid nodule lesions are benign while approximately $20 \%$ are malignant. Most of the malignant lesions in the thyroid are papillary thyroid carcinoma (PTC), followed by follicular, medullary, and anaplastic thyroid carcinoma (5). On CT or MRI, malignant nodules do not have specific imaging findings $(6,7)$. PTC $\leq 1 \mathrm{~cm}$ is defined as papillary thyroid micro-carcinoma (PTMC), and it is the most prevalent type of papillary thyroid carcinoma. A diameter of $1 \mathrm{~cm}$ is regarded as the cut-off above which US examination is suggested for incidental thyroid nodule (1).

In the present study, we examined factors including frequency, size, age, and sex with regard to the incidental finding of thyroid nodules in patients presenting for oral and maxillofacial CT examination. Additionally, we conducted a retrospective review of patients without a history of thyroid disease who underwent CT examination in our institution between January 2009 and December 2009 for oral and maxillofacial pathology with incidental findings in the thyroid.

\section{Case report}

A 59-year-old man was referred from a private dental practice. He had undergone an extraction of first molar of right mandible for spontaneous pain. Following the extraction, the symptoms persisted, and he was referred to our university hospital. A panoramic radiograph showed a multiloculated radiolucency extending from the third molar area of the right mandible extending to the mandibular ramus. There was no root resorption of the second molar (Fig. 1). CT images demonstrated low density in the multiloculated area with lingual expansion. The interior of the lesion was of heterogeneous soft tissue density that did not undergo contrast enhancement (Fig. 2). MR images showed intermediate signal intensity (SI) on T1WI, high to markedly high SI on STIR and strong enhancement of the lesion's wall and weak enhancement inside the lesion 


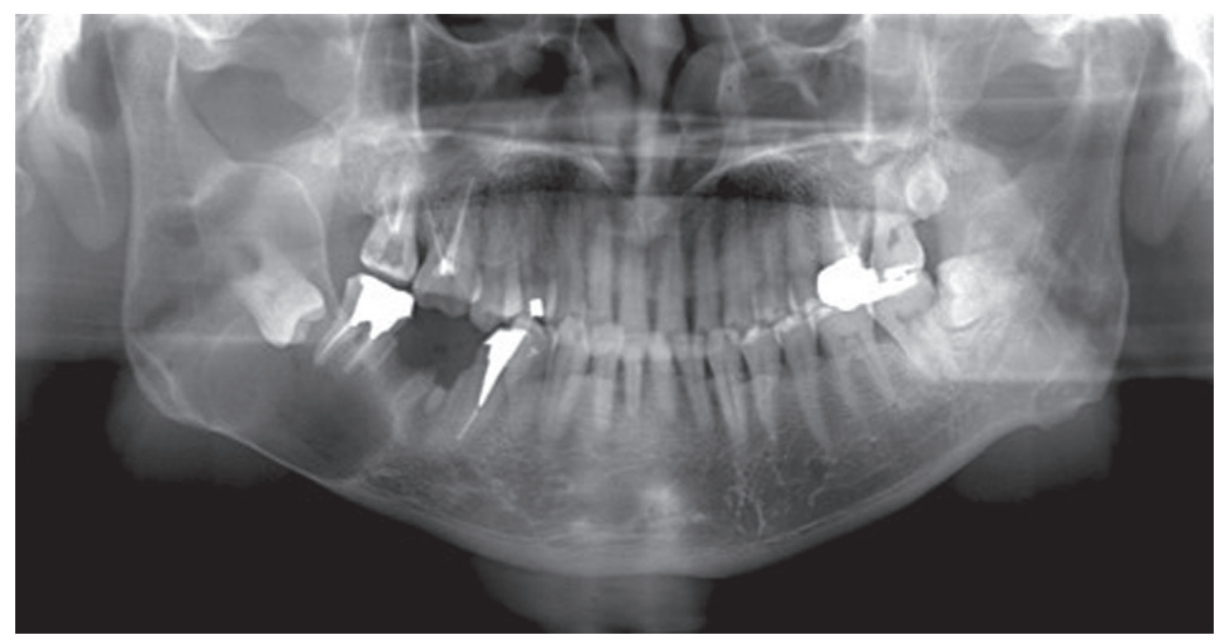

Figure 1. Panoramic image, multilocular lesion on right side area of mandible from first molar area extended to ramus mandible area and third molar was inside the lesion.

A

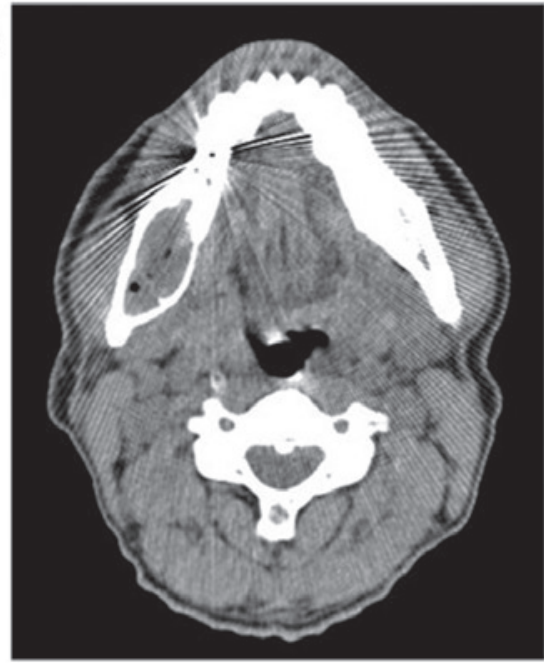

B

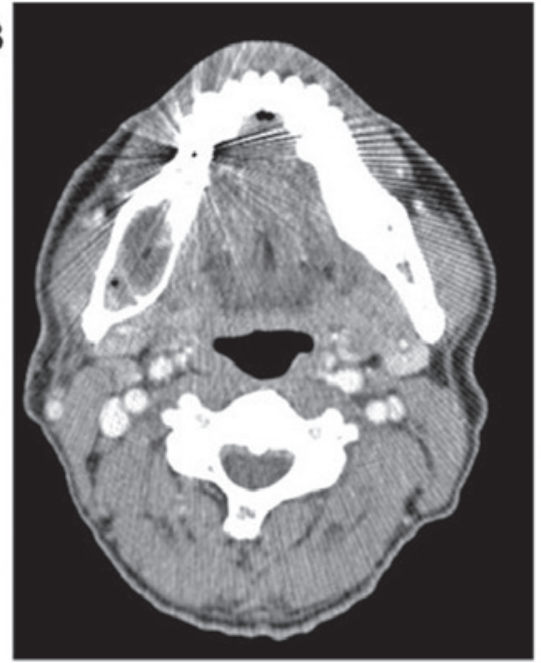

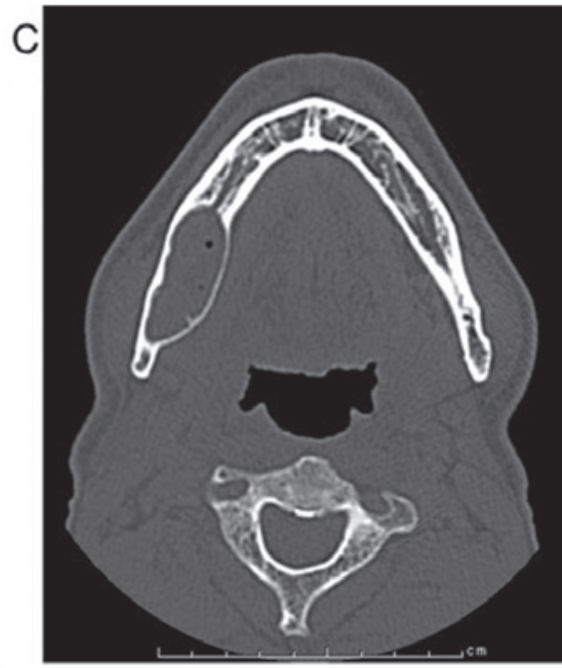

Figure 2. CT images of mandibular lesion. (A) Axial soft tissue CT images show multilocular lesion with clear boundary, low and several low concentration areas, which seems mixed with air, (B) axial contrast-enhancement demonstrated heterogeneous area inside the lesion, and (C) axial bone CT image showing bone was bulging on the lingual side.
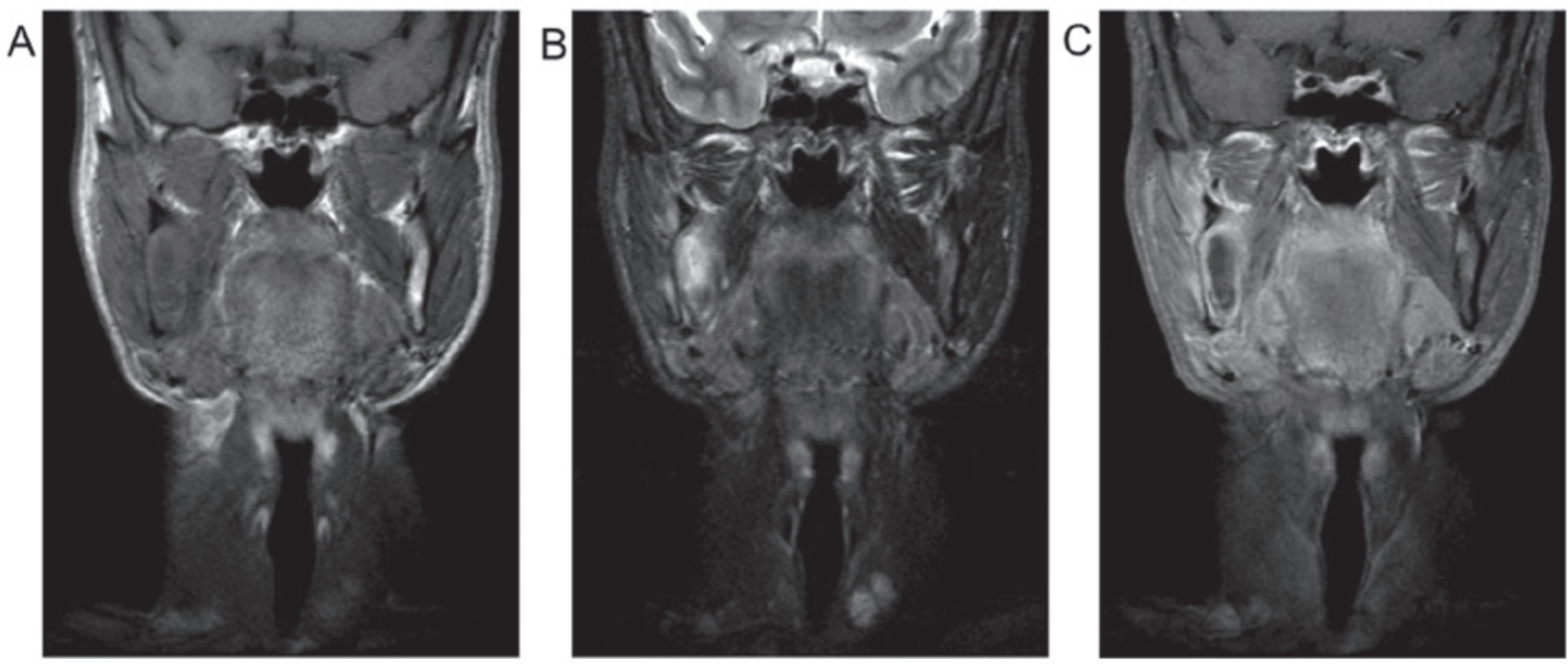

Figure 3. MR images of mandibular lesion. (A) T1WI, homogeneous low to slightly high SI. (B) STIR showed high to markedly high SI indicated cystic area inside the lesion. (C) CE-T1WI demonstrated tight strong enhancement of wall and weak enhancement inside the lesion. 

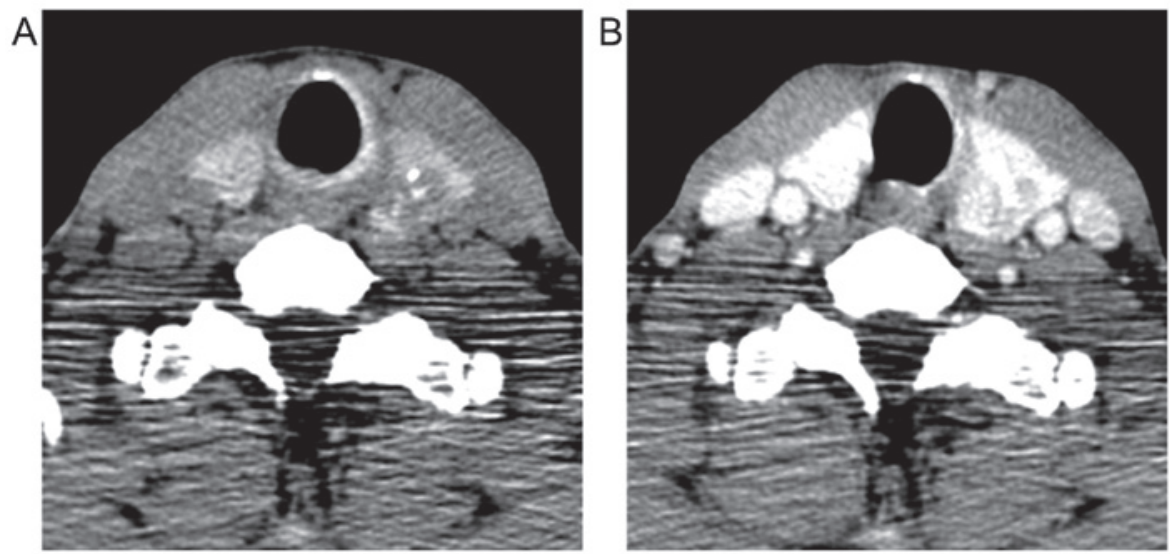

Figure 4. CT images on thyroid level. (A) Soft tissue CT image shows left lobe was enlarged. (B) CE-CT demonstrated lesion with calcification inside, lesion showed non-uniform low concentration with approximately $28 \mathrm{~mm}$ in diameter size.
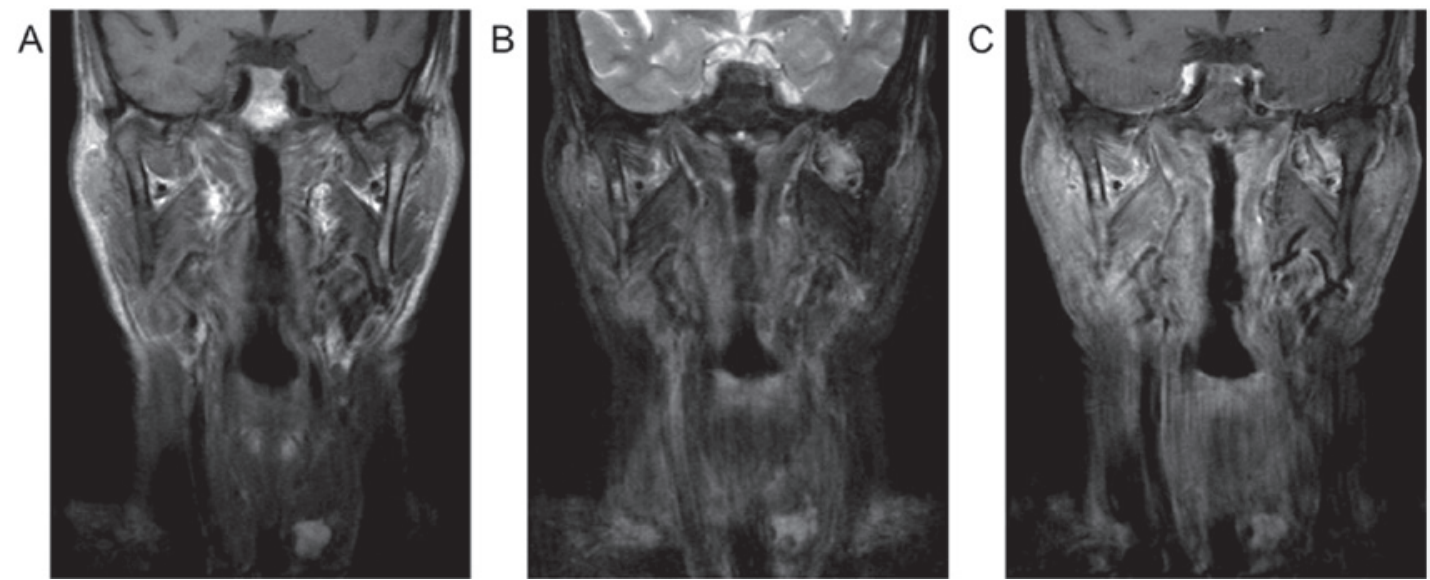

Figure 5. MR images of thyroid lesion. (A) T1WI image shows high signal intensity, (B) STIR image demonstrated high signal intensity, and (C) CE-T1WI shows strong enhancement.
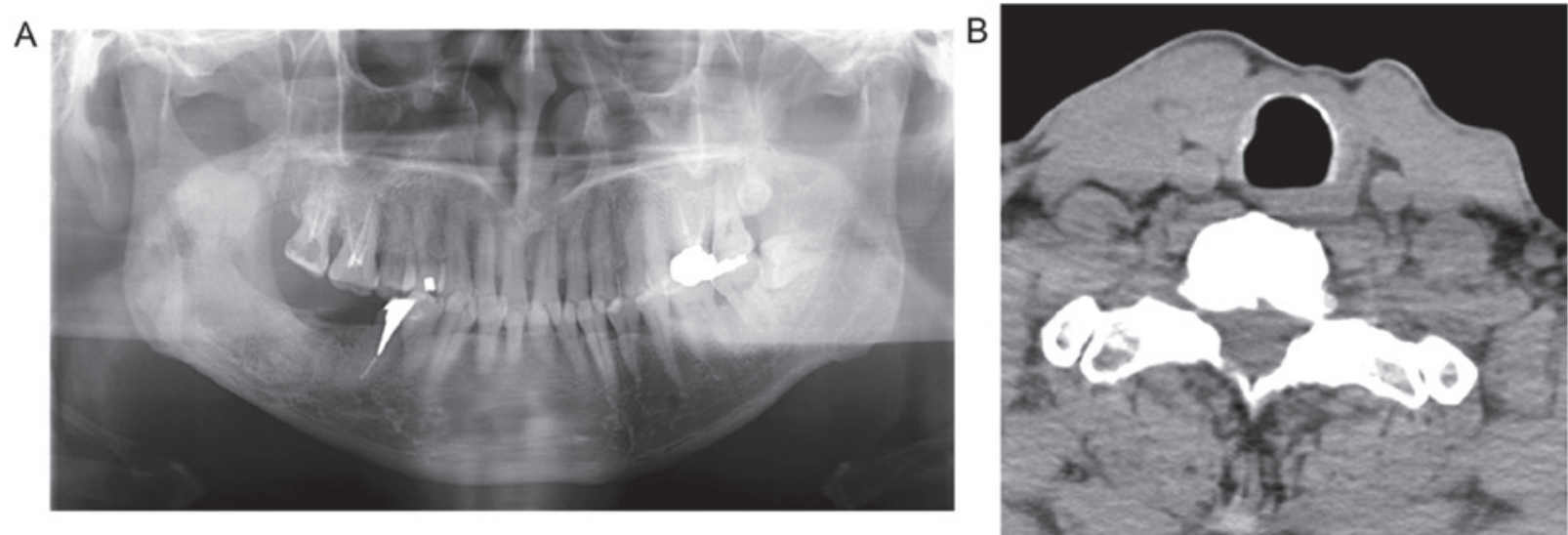

Figure 6. Follow up of patient. (A) Panoramic image shows no recurrence of the mandibular lesion and (B) CT image 10 years after total thyroidectomy shows no recurrence lesion on thyroid area.

(Fig. 3). Based on these findings, a preliminary diagnosis of keratocystic odontogenic tumor was made.

On the same CT images, the left lobe of the thyroid was enlarged by a $28-\mathrm{mm}$ nodule containing calcifications. It showed non-uniform low density, and heterogeneous contrast enhancement (Fig. 4). MR images demonstrated high SI on T1WI and STIR and strong enhancement on CE-T1WI (Fig. 5).

The patient was referred to the Department of Breast and Thyroid Surgery. Fine-needle aspiration biopsy (FNAB) of the 
thyroid nodule suggested category V (suspicious for malignancy) based on the Bethesda System for reporting thyroid cytopathology. He was treated with a total thyroidectomy and bilateral neck dissection. The histological diagnosis of the surgical specimen was papillary thyroid carcinoma. A CT performed 3 months after surgery showed no evidence of recurrence (Fig. 6). After the patient's recovery from surgery, fenestration was performed on the right mandibular lesion. After 3 years, panoramic images showed no lesion recurrence in the mandible. Ten years post-thyroidectomy, the patient is well with no evidence of recurrence.

Retrospective review. In total, 169 patients, 87 males and 82 females, with a mean age of 67.3 years (range, 17-92 years), as well as 65 patients (38.5\%), 43 (66.2\%) female and 22 (33.8\%) male had abnormal findings in the thyroid for one year.

\section{Discussion}

Use of advance radiology devices in oral and maxillofacial region such as US, CT, MRI and PET-CT could produce high quality examination of the maxillofacial region and contiguous structures and organs. Based on the increase of quality and usage of the devices, incidental findings in USG, CT and MRI examination are often found.

Incidental finding is an unexpected discovery rather than the initial purpose for which the radiographic examination was carried out. Incidental findings may be anatomy disorder or neoplasm, most of which are asymptomatic and insidious. In head and neck, the most common area of incidentaloma is thyroid (8). Thyroid nodules appear with nodular or rim calcification on CT images and may likely be malignant (3). However, unlike US which has adequate spatial resolution such as microcalcification or margin detail, CT and MR images have no indicators to differentiate between benign and malignant tumors $(6,7)$. Both benign and malignant tumors demonstrated intermediate signal intensity on T1WI and high signal intensity on T2WI, even if in our case showed high signal intensity in T1WI and STIR. Additional investigations are required to determine malignancy such us US or FNA (7,9-11).

Papillary thyroid carcinoma is the most common malignant tumor in thyroid. Some risk factors of PTC are associated with ionizing radiation exposure, particularly head and neck or total body irradiation for bone marrow transplantation and family history of thyroid carcinoma (12). The PTC with less than $5 \mathrm{~mm}$ in diameter has almost no metastatic potential. On PTC case size and patient age are important for treatment consideration. Some study of PTC has grouping PTC based on size $(2,7,10,11,13)$. PTC with $<5 \mathrm{~mm}$ known as papillary thyroid microcarcinoma (PTMC), in elderly patients, progressivity of lesion was not evident during observation and immediate surgery was not needed $(4,11,13)$.

As age is one of the considerations for PTC treatment, clinicians tend to focus on younger patients $(7,10)$ as progression of the disease is more likely in younger patients compared to elderly patient $(2,13)$. In addition, the ratio of malignant and benign was higher in younger patients, although age was not an independent predictor of PTMC $(10,11,13)$.

Noguchi et al reported that thyroid PTMC prevalence was higher in women (11), similar with our retrospective survey, which showed a predominance of cases in females, although some authors reported that sex was not an independent predictor of PTMC, as the high incidence of PTMC in women was due to more frequent exposure to diagnostic or treatment procedures (10). Radiologists have an important role to determine incidental finding and provide a complete report and to be aware about the potential of malignancy (4). Tanpitukponse et al reported only a small proportion of incidental finding of thyroid lesions from CT and MRI examinations that were referred from radiologists to clinicians, had undergone additional examination (14). Patient age and nodule size influenced clinicians to make decisions regarding additional examination $(6,11,13-15)$. Hoang et al reported that $2 \mathrm{~cm}$ is the threshold size of CT and MR examinations for incidental finding of thyroid lesion (6). Other factors that should be considered include patient comorbid condition or financial condition. In addition, different perceptions between radiologists and clinicians towards incidental finding pose a potential problem. Therefore, collaboration between radiologists and clinicians is imperative in the incidental finding of thyroid lesion.

In summary, the wider usage of $\mathrm{CT}$ and MR devices on head neck examination increases the incidental finding of PTC. Treatment consideration and prognosis of incidental finding of PTC depend on nodule size and patient age. Collaboration between radiologists and clinicians is important in the incidental finding of PTC.

\section{References}

1. Johnson PT, Horton KM, Megibow AJ, Jeffrey RB and Fishman EK: Common incidental findings on MDCT: Survey of radiologist recommendations for patient management. J Am Coll Radiol 8: 762-767, 2011.

2. Nguyen XV, Choudhury KR, Eastwood JD, Lyman GH, Esclamado RM, Werner JD and Hoang JK: Incidental thyroid nodules on CT: Evaluation of 2 risk-categorization methods for work-up of nodules. AJNR Am J Neuroradiol 34: 1812-1817, 2013.

3. Yoon DY, Chang SK, Choi CS, Yun EJ, Seo YL, Nam ES, Cho SJ, Rho YS and Ahn HY: The prevalence and significance of incidental thyroid nodules identified on computed tomography. J Comput Assist Tomogr 32: 810-815, 2008.

4. Hoang JK, Branstetter BF IV, Gafton AR, Lee WK and Glastonbury CM: Imaging of thyroid carcinoma with CT and MRI: Approaches to common scenarios. Cancer Imaging 13: 128-139, 2013.

5. Rosenkrantz AB: Differences in perceptions among radiologists, referring physicians, and patients regarding language for incidental findings reporting. AJR Am J Roentgenol 208: 140-143, 2017.

6. Hoang JK, Langer JE, Middleton WD, Wu CC, Hammers LW, Cronan JJ, Tessler FN, Grant EG and Berland LL: Managing incidental thyroid nodules detected on imaging: White paper of the ACR Incidental Thyroid Findings Committee. J Am Coll Radiol 12: 143-150, 2015.

7. Shetty SK, Maher MM, Hahn PF, Halpern EF and Aquino SL: Significance of incidental thyroid lesions detected on CT: Correlation among CT, sonography, and pathology. AJR Am J Roentgenol 187: 1349-1356, 2006.

8. American Thyroid Association (ATA) Guidelines Taskforce on Thyroid Nodules and Differentiated Thyroid Cancer; Cooper DS, Doherty GM, Haugen BR, Kloos RT, Lee SL, Mandel SJ, Mazzaferri EL, McIver B, Pacini F, et al: Revised American Thyroid Association management guidelines for patients with thyroid nodules and differentiated thyroid cancer. Thyroid 19: 1167-1214, 2009.

9. Hoang JK, Lee WK, Lee M, Johnson D and Farrell S: US Features of thyroid malignancy: Pearls and pitfalls. Radiographics 27 : 847-865, 2007. 
10. Slijepcevic N,Zivaljevic V, Marinkovic J, Sipetic S, Diklic A and Paunovic I: Retrospective evaluation of the incidental finding of 403 papillary thyroid microcarcinomas in 2466 patients undergoing thyroid surgery for presumed benign thyroid disease. BMC Cancer 15: 330, 2015.

11. Noguchi S, Yamashita H, Uchino S and Watanabe S: Papillary microcarcinoma. World J Surg 32: 747-753, 2008.

12. Xu L, Li G, Wei Q, El-Naggar AK and Sturgis EM: Family history of cancer and risk of sporadic differentiated thyroid carcinoma. Cancer 118: 1228-1235, 2012.

13. Ito Y, Miyauchi A, Kihara M, Higashiyama T, Kobayashi K and Miya A: Patient age is significantly related to the progression of papillary microcarcinoma of the thyroid under observation. Thyroid 24: 27-34, 2014.
14. Tanpitukpongse TP, Grady AT, Sosa JA, Eastwood JD, Choudhury KR and Hoang JK: Incidental thyroid nodules on CT or MRI: Discordance between what we report and what receives workup. AJR Am J Roentgenol 205: 1281-1287, 2015.

15. Tufano RP, Noureldine SI and Angelos P: Incidental thyroid nodules and thyroid cancer: Considerations before determining management. JAMA Otolaryngol Head Neck Surg 141: 566-572, 2015. 